\title{
JNK-NQ01 axis drives TAp73-mediated tumor suppression upon oxidative and proteasomal stress
}

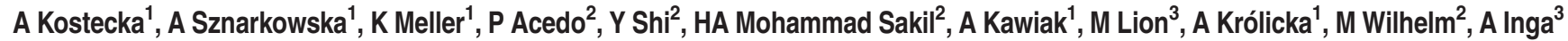 \\ and J Zawacka-Pankau*, ${ }^{\star, 1,2}$
}

Hyperproliferating cancer cells produce energy mainly from aerobic glycolysis, which results in elevated ROS levels. Thus aggressive tumors often possess enhanced anti-oxidant capacity that impedes many current anti-cancer therapies. Additionally, in ROS-compromised cancer cells ubiquitin proteasome system (UPS) is often deregulated for timely removal of oxidized proteins, thus enabling cell survival. Taken that UPS maintains the turnover of factors controlling cell cycle and apoptosis - such as p53 or p73, it represents a promising target for pharmaceutical intervention. Enhancing oxidative insult in already ROS-compromised cancer cells appears as an attractive anti-tumor scenario. TAp73 is a bona fide tumor suppressor that drives the chemosensitivity of some cancers to cisplatin or $\gamma$-radiation. It is an important drug target in tumors where p53 is lost or mutated. Here we discovered a novel synergistic mechanism leading to potent $\mathrm{p} 73$ activation and cancer cell death by oxidative stress and inhibition of $20 \mathrm{~S}$ proteasomes. Using a small-molecule inhibitor of $20 \mathrm{~S}$ proteasome and ROS-inducer - withaferin A (WA), we found that WAinduced ROS activates JNK kinase and stabilizes phase II anti-oxidant response effector NF-E2-related transcription factor (NRF2). This results in activation of Nrf2 target - NQ01 (NADPH quinone oxidoreductase), and TAp73 protein stabilization. The observed effect was ablated by the ROS scavenger - NAC. Concurrently, stress-activated JNK phosphorylates TAp73 at multiple serine and threonine residues, which is crucial to ablate TAp73/MDM2 complex and to promote TAp73 transcriptional function and induction of robust apoptosis. Taken together our data demonstrate that ROS insult in combination with the inhibition of 20S proteasome and TAp73 activation endows synthetic lethality in cancer cells. Thus, our results may enable the establishment of a novel pharmacological strategy to exploit the enhanced sensitivity of tumors to elevated ROS and proteasomal stress to kill advanced tumors by pharmacological activation of TAp73 using molecules like WA.

Cell Death and Disease (2014) 5, e1484; doi:10.1038/cddis.2014.408; published online 23 October 2014

Targeted therapeutics such as kinase inhibitors have emerged as promising drugs for cancer patients but as for the chemotherapy regimes, drug resistance, often linked to oncogenic reprogramming of cancer cells, confines the effectiveness of these therapies. ${ }^{1-3}$

Redox homeostasis is essential to sustain cellular functions and enable cell survival. In normal cells, modest reactive oxygen species (ROS) modulates many cellular processes through stress-kinase pathways, including mitogen-activated protein kinase (MAPK) pathway. ${ }^{4}$ Interestingly, ROS is emerging as master regulators of embryonic stem cells' renewal, proliferation, immune response and differentiation. ${ }^{5-8}$ Increased oxidative stress burden, often linked to the chronic inflammation, is implicated in the tumorigenesis and leads to the elevated anti-oxidative response program in stress-adapted tumors. As a result, it often confers chemoresistance to the ROS-driven treatments. ${ }^{9-13}$ Based on this knowledge, a new therapeutic concept is being developed that focuses on exploiting master regulators of the anti-oxidant response, such as NRF2, to overcome the tumor resistance. ${ }^{11}$

In normal cells, elevated levels of ROS often promote an enhanced activity of the proteasome system to remove the oxidized proteins, thus to prevent their toxicity. This phenomenon is observed in many human neurodegenerative diseases, including Alzheimer's disease, Parkinson's disease and Huntington's disease. ${ }^{14-19}$ Similarly, ROS-compromised cancer cells, for timely removal of oxidized proteins, possess an enhanced system for the protein ubiqutination and an amplified activity of the ubiquitin proteasome pathway (UPP), in particular $20 \mathrm{~S}$ proteasome particle. ${ }^{14}$ The $20 \mathrm{~S}$ proteasome particle is additionally important for the degradation of the liable tumor suppressors like p53 and TAp73, the process rescued by NADPH quinone oxidoreductase (NQO1). ${ }^{20}$ With the approval of bortezomib, to treat multiple myeloma, inhibition of the proteasome activity emerges as a promising anti-cancer therapy. ${ }^{21}$

Withaferin A (WA) and withanone (WN) are steroidal lactones obtained from Withania sommnifera - medical herb

\footnotetext{
${ }^{1}$ Department of Biotechnology, Intercollegiate Faculty of Biotechnology, University of Gdansk and Medical University of Gdansk, Gdansk, Poland; ${ }^{2}$ Department of Microbiology, Tumor and Cell Biology, Karolinska Institutet, Stockholm, Sweden and ${ }^{3}$ Centre for Integrative Biology, CIBIO, University of Trento, Mattarello, Italy

*Corresponding author: J Zawacka-Pankau, Department of Microbiology, Tumor and Cell Biology, Karolinska Institutet, Nobels vag 16, Stockholm 171 77, Sweden. Tel: +46 8524 86311; Fax: +46 831 1101; E-mail: joanna.zawacka-pankau@ki.se or joanna.zawacka-pankau@ biotech.ug.edu.pl

Abbreviations: ROS, reactive oxygen species; PI, proteasome inhibitor; WA, Withaferin A; MAPK, mitogen-activated protein kinase; UPP, ubiquitin proteasome pathway; IP, immunoprecipitation; Nrf2, NF-E2-related transcription factor; NAC, N-acetyl-L-cysteine; NQO1, NADPH quinone oxidoreductase; NAC, N-acetyl-L-cysteine; JNK, Jun N-terminal kinase; NHDF, normal human diploid fibroblasts; MEF, mouse embryonic fibroblasts; HMOX-1, heme oxygenase 1; iASPP, inhibitor of apoptosis-stimulating protein of $p 53$

Received 03.5.14; revised 24.7.14; accepted 18.8.14; Edited by G Melino
} 
of Ayurveda. ${ }^{22}$ They possess anti-inflammatory and anticancer properties. WA inhibits catalytic $\beta$-subunit of 205 proteasome core particle similarly to the clinically used proteasome inhibitor $(\mathrm{PI})$ bortezomib ${ }^{23-26}$ and was shown to impede NF- $k B$ function in cancer cells ${ }^{27}$ via stabilization of $I_{\kappa} \mathrm{B} a$, the cytoplasmic inhibitor of NF- $\kappa \mathrm{B}$. Similarly, withanolide $\mathrm{D}$ inhibited degradation of $\mathrm{I}_{\kappa} \mathrm{B} a$ by targeting the UPP. ${ }^{28}$ Anti-tumor functions of WA were also linked to its direct interactions with annexin $11{ }^{29}$ anti-angiogenic activity, ${ }^{28}$ inhibition of STAT-1/3 and Akt signaling pathways and subsequent stabilization of p53 by activated ARF, which inactivates MDM2 and prevents its binding to p53. ${ }^{30}$ Taken together, WA is a potent inhibitor of tumorigenesis both in vitro and in vivo, but the exact mechanism underlying WA anticancer activity remains unresolved.

Pharmacological recapitulation of p53 activity is currently exploited as a new therapeutic strategy to combat cancers. ${ }^{31}$ Several p53 reactivating small molecules have been developed and are currently undergoing preclinical or clinical developments. ${ }^{31,32}$ Their implementation in clinics, mainly due to the adverse pharmacokinetics, is still impeded. This, together with the fact that p53 is lost or mutated in $>50 \%$ of all human cancers, indicates that novel approaches are needed for the effective tumor killing.

TP73 belongs to the p53 family of genes, including TP63 and TP53. The role of $\mathrm{p} 63$ and $\mathrm{p} 73$ in cancer is determined by the existence of two classes of isoforms, TA (transcriptionally active) and $\Delta \mathrm{N}$ (transcriptionally inactive $\mathrm{N}$ terminus deleted). TA isoforms structurally resemble p53 and act as tumor suppressors. ${ }^{33} \mathrm{p} 53$, TAp63 and TAp73 can transactivate many of the same target genes and execute their tumor-suppressor function by guarding the genomic stability and promoting cell cycle arrest, replicative senescence or apoptosis ${ }^{34-36}$ Around $70 \%$ of $\mathrm{TAp} 73^{-/-}$mice develop tumors (mostly lung adenocarcinomas), and the rest show premature aging linked to the deregulated metabolism. ${ }^{37}$ This indicates that TAp73 is a tumor suppressor and important regulator of metabolism. Unlike TP53, which is mutated in about $50-80 \%$ of human cancers, the TP73 gene mutations are rare. ${ }^{34}$ Therefore TA isoforms of $\mathrm{p} 73$ can compensate for p53 function in tumors with lost or mutated p53.

In cancer cells, the transcriptional activity of TAp73 is kept in check by several protein inhibitors, such as $\Delta N p 73, \Delta N p 63$, iASPP $^{38}$ and aurora kinase $A,{ }^{39}$ and mutant p53 form heterocomplexes with TAp73 and abrogate its transcriptional activity. ${ }^{33,34,38-40}$ MDM2, E3 ubiquitin ligase that binds p53 and promotes its ubiquitin-dependent degradation, blocks TAp73 transcriptional activity via direct binding to its transactivation domain but does not promote TAp73 proteolytic disassembly. Similar to p53, TAp73 can be directed for degradation in an ubiquitin-independent manner, which is mediated by $20 S$ proteasomes. ${ }^{20}$ Asher et al. ${ }^{20}$ reported that NQO1 protein directly interacts with TAp73a via its SAM domain, and protects it from the proteasomal cleavage. ${ }^{20}$ IR-mediated DNA damage and oncogenic insult both activate TAp73 by releasing it from its negative regulators, such as MDM2, MDMX or iASPP. ${ }^{34,38}$ We and others have shown that TAp73 can serve as a therapeutically relevant target of anticancer molecules, including Nutlin, ${ }^{41}$ 37AA peptide, ${ }^{42}$ RETRA $^{43}$ and protoporphyrin IX. ${ }^{44}$ This implies the 'druggable' nature of TAp73 protein however, solid reports supporting the prominent, tumor-suppressive outcome of TAp73 restoration are still missing.

Selivanova and colleagues ${ }^{45}$ have previously shown that small-molecule RITA, a known p53 activator, promotes p53-mediated cell death in cancer cells by synthetic lethal mechanism converging on concurrent inhibition of thioredoxin reductase (TrxR), which results in elevated oxidative stress and inhibition of p53/MDM2 complex. This amends p53 from growth suppressor to effective apoptosis inducer. ${ }^{46}$ In the present study, we discovered that ROS insult is indispensable for an efficient induction of apoptosis by TAp73 upon treatment with proteasomal inhibitor - WA. This is a new direction in the field of pharmacological modulation of p73 pathway for efficient tumor killing that can be further exploited to develop potent anti-cancer agents, such as WA.

\section{Results}

Cell death is detected in tumor cells with TP53 gene deletion but not in normal cells after WA treatment. WA (for structure, refer to Supplementary Figure S1A) was shown to exert anti-tumor activity in cells expressing mutant or wildtype p53. ${ }^{29,47}$ To address the question whether cancer cells lacking p53 but expressing TA isoform of p73 are dying upon treatment with WA, we performed viability and clonogenic assays in $\mathrm{H} 1299$, human lung adenocarcinoma cells and in HCT $116^{\text {TP53-I- }}$ human colon cancer cell line previously engineered for homozygous deletion of TP53 gene resulting in p53 protein loss of function. ${ }^{48}$ As the median inhibition concentration of $50 \%\left(\mathrm{IC}_{50}\right)$ was $0.79 \mu \mathrm{M}$ in $\mathrm{H} 1299(48 \mathrm{~h})$ as compared with $3.9 \mu \mathrm{M}$ in fibroblasts (normal human dermal fibroblasts (NHDF)) (Supplementary Figure S1B), we applied 0.5 and $1 \mu \mathrm{M}$ WA in our studies. Clonogenic survival assay revealed significant decrease in cell number already at low doses of WA in H1299 cells (Figure 1a, Supplementary Figure S6A), and $1 \mu \mathrm{M}$ was effective in both cell lines tested. This correlated with the increased levels of active caspases (Figure 1b), while the use of the pan-caspase inhibitor zVAD-FMK partially reversed WA-mediated inhibition of cell proliferation (Figure 1c, Supplementary Figure S6B). Consistent with caspase activation, WA induced SubG1 cell population in treated cells, which regressed after zVAD, a pan caspase inhibitor, pretreatment (Figure 1d).

Upon stresses such as DNA damage or oncogene activation, TAp73, similarly to p53, regulates transcription of apoptotic genes, including PUMA, NOXA and $B A X^{34} \mathrm{We}$ found that WA promoted stabilization of TAp73 at the protein level, which correlated with the induction of cleaved PARP p85 fragment indicating that cells were dying of apoptosis (Figures $1 \mathrm{e}$ and $\mathrm{f}$ ). Western blotting analysis revealed that the increase in TAp73 protein levels in cancer cells correlates with the elevated protein levels of PUMA, Bax and Bid. qPCR analysis shows that mRNA levels of TAp73 and $\triangle N p 73$ were not significantly affected upon WA (Supplementary Figures S1C and D), while we observed upregulation of TAp73 pro-apoptotic target PUMA and NOXA and downregulation of $\mathrm{BCl}-2$ (Figure $2 \mathrm{f}$ ). 


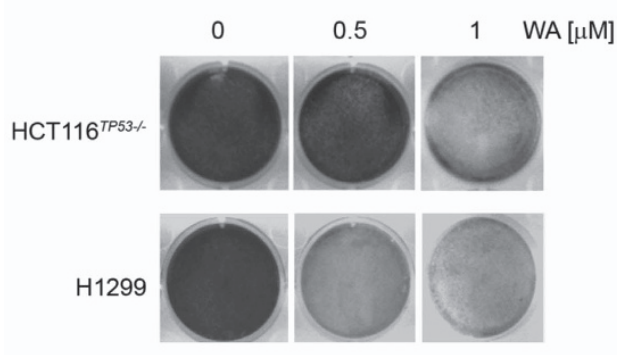

c
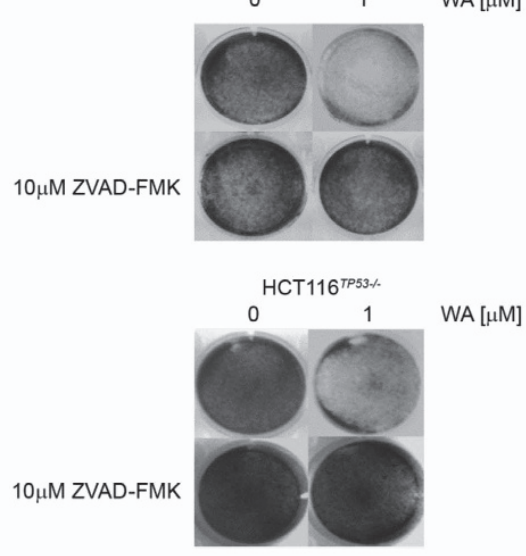

e

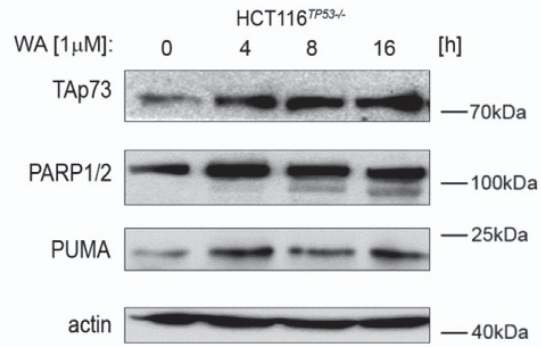

g

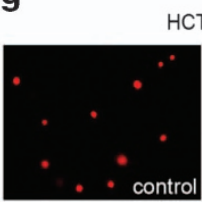

HCT116 ${ }^{\text {TP53\% }}$ b

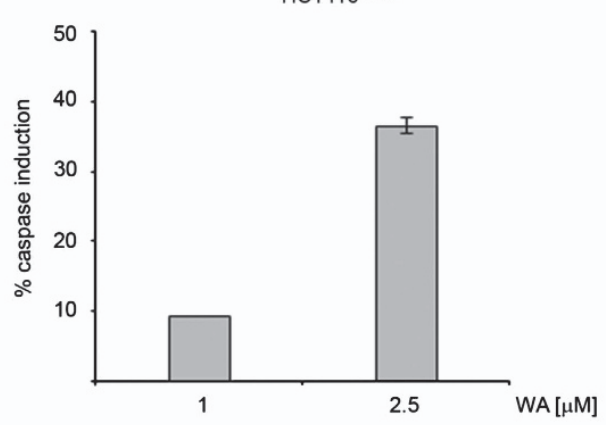

d

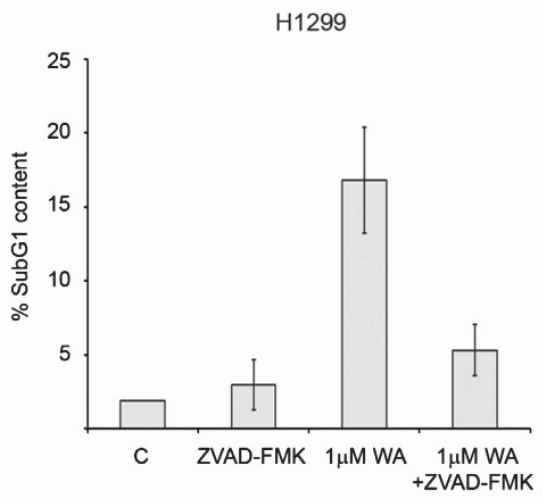

f

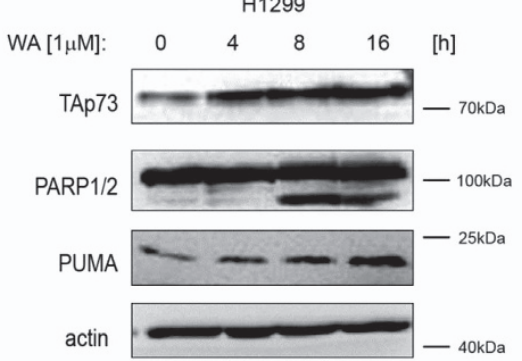

i

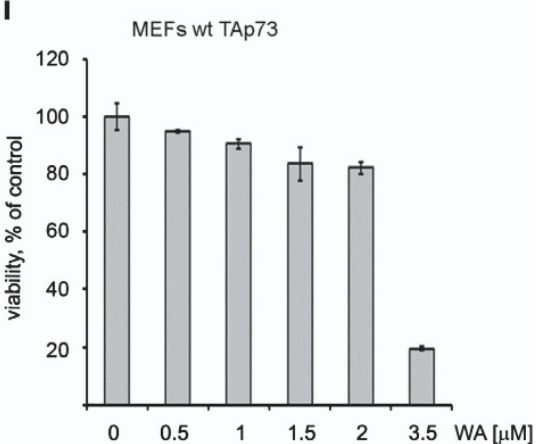

Figure 1 WA promotes apoptosis in cancer cells lacking p53. (a) H1299 and HCT116 ${ }^{T P 53-l-}$ cells were treated with WA for $48 \mathrm{~h}$ and stained with crystal violet for clonogenic survival determination. (b) Growth inhibition correlated with caspase activation by WA. (c) Caspase inhibitor ZVAD-FMK protects H1299 and HCT116 TP53-I- from growth inhibition by WA. (d) WA stimulates accumulation of $\mathrm{H} 1299$ in subG1 fraction, which is reversed by zVAD-FMK pretreatment. (e and f) Immunoblots presenting induction of TAp73 in HCT116 ${ }^{\text {TP53- } I-}$ and in $\mathrm{H} 1299$ cells, which coincides with the induction of apoptotic PUMA and with the PARP cleavage. (g) HCT116 $6^{\text {TP53-I- }}$ cells after treatment with WA or $\mathrm{H}_{2} \mathrm{O}_{2}(100 \mu \mathrm{M})$ for $3 \mathrm{~h}(\times 100$ magnification) $(n=3)$. (h) Immunoblot of WA-treated NHDF presenting TAp73, TAp63 and BAX protein levels. (i) WST-1 assay performed in MEFs wt TAp73 treated with WA 


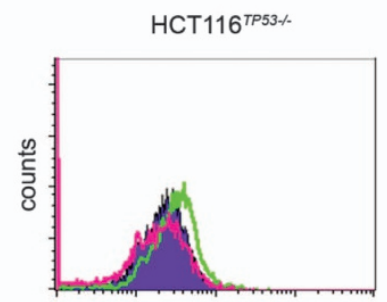

FL1-H

control

- $1 \mu \mathrm{M} W \mathrm{NA}$

- $1 \mu M W A+N A C$

C

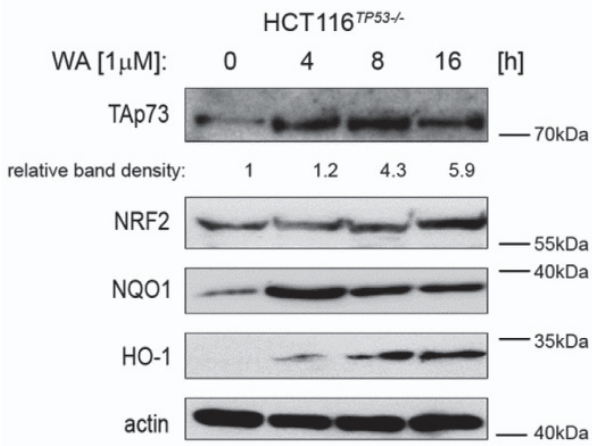

e

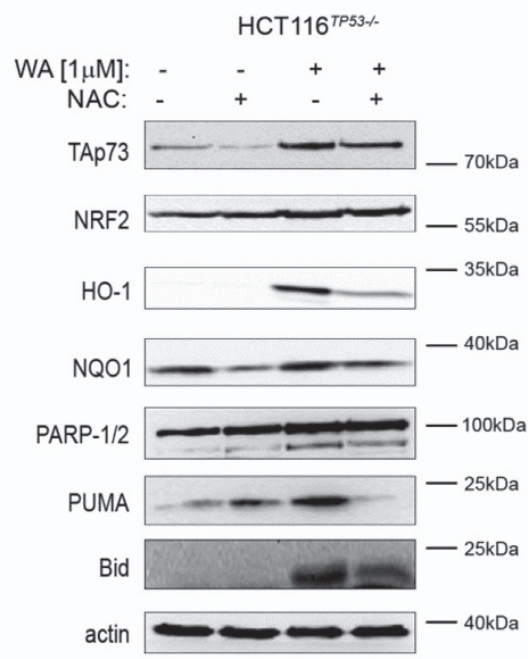

b
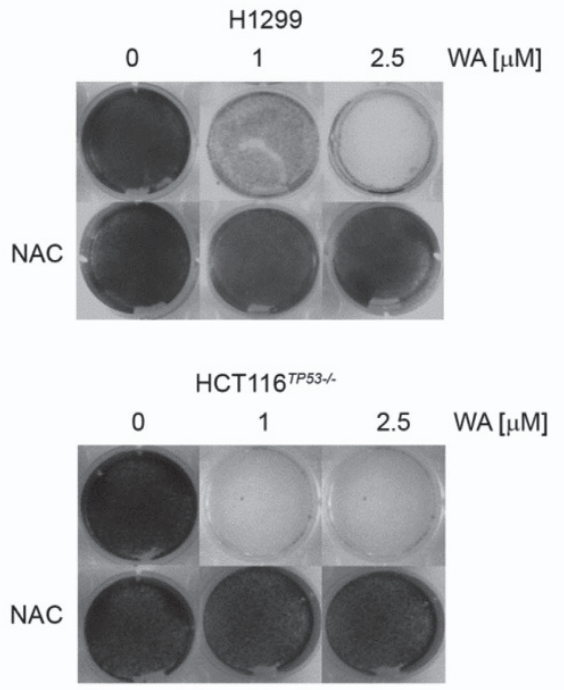

d

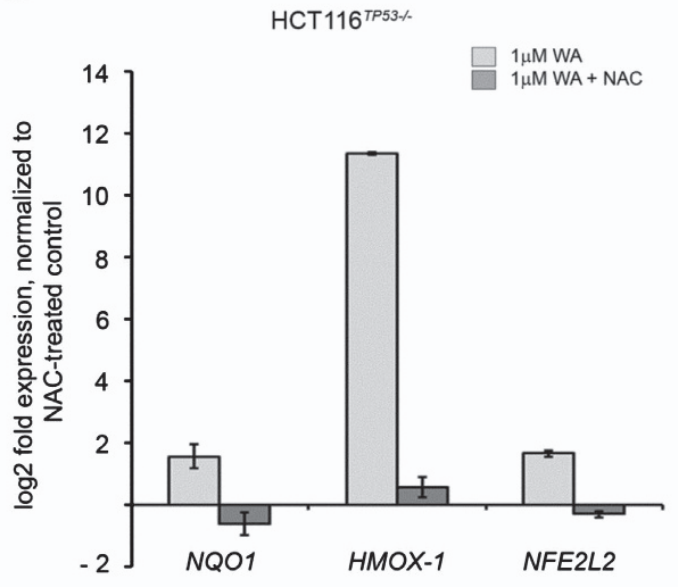

f

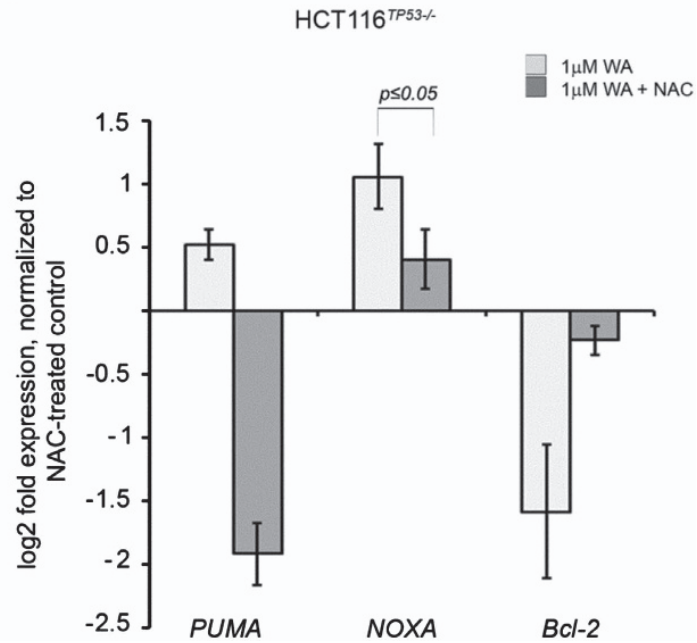

Figure 2 WA induces ROS and anti-oxidant response in tumor cells to trigger cell death. (a) DCF-DA-stained H1299 cells show elevated ROS levels upon WA treatment, which was prevented by NAC pretreatment. (b) ROS scavenger NAC abrogates WA-induced growth suppression in H1299 and HCT116 ${ }^{\text {TP53-I- }}$ cells. (c) WA-induced ROS upregulates protein levels of TAp73 and PUMA along with NRF2 and its anti-oxidant effectors NQO1 and HO-1. (d) ROS induced the expression of anti-oxidant response genes NFE2L2, NQO1 and HMOX-1 whereas NAC pretreatment reversed this effect. (e) Immunoblots of WA-treated HCT116 ${ }^{\text {TP53-l- }}$ cells show induced TAp73 protein levels, which correlated with the induction of NRF2, NQ01, HO-1, PUMA, Bid and PARP. This effect was prevented by NAC pretreatment for $8 \mathrm{~h}$ that is also apparent from (f) $\mathrm{qPCR}$ analysis 
As the systemic toxicity of the existing anti-cancer drugs limits their broad application, we assessed the genotoxicity of WA in cancer cells and in NHDF. The use of human normal cells in toxicity studies is of key relevance when planning future clinical trials for new anti-cancer drugs. Alkaline comet assay implies that WA did not promote DNA-damage (Figure 1g, Supplementary Figure S1E), did not affect viability of NHDF at concentrations tested (Supplementary Figure S1B) and did not induce TAp73 and pro-apoptotic proteins in normal cells (Figure 1h). What is more, we could not detect toxicity of WA toward primary mouse embryonic fibroblasts (MEFs) at the concentrations tested (Figure 1i).

Taken together, we conclude that WA is a non-genotoxic agent that promotes cell death in tumor cells, which coincides with the increase of TAp73 protein levels, accumulation of apoptotic proteins PUMA and NOXA and cleaved PARP.

WA induces oxidative stress, which facilitates TAp73 activation and inhibits proliferation of cancer cells. Hahm et al. ${ }^{47}$ described that WA inhibits oxidative phosphorylation, which is a consequence of the generation of superoxide due to mitochondrial dysfunction. To address the question whether the elevation of ROS by WA is cell line specific or not, we measured the generation of hydroxyl, peroxyl and other ROS by employing cell-permeable reagent $2^{\prime}, 7^{\prime}$-dichlorofluorescein diacetate (DCF-DA) and measuring its fluorescence upon activation. Our experiments provide the evidence that WA effectively induced ROS in cancer cell lines deprived of p53 (Figure 2a, Supplementary Figure S2A). Pretreatment of cells with ROS scavenger $\mathrm{N}$-acetyl-L-cysteine (NAC) inhibited accumulation of ROS by WA and ablated the anti-proliferative effect of WA in $\mathrm{H} 1299$ and HCT $116^{\text {TP53-I- }}$ cells (Figures $2 a$ and $b$ and Supplementary Figure S2A, and S6C and D).

In cells, oxidative stress activates effector proteins belonging to the anti-oxidant system response, including the master regulator - NRF2. Thus, next we investigated the downstream effectors of ROS triggered by WA and how they contribute to cancer cell death. As ROS triggers the anti-oxidant response, we sought to investigate whether WA activates NRF2 as a consequence of the induced oxidative stress. ${ }^{49}$ In our settings, we found that WA only slightly induced mRNA levels of NRF2 and efficiently promoted NRF2 stabilization on the protein level. It correlated with a potent overexpression of NRF2 target genes NQO1 and heme oxygenase 1 (HMOX-1 or HO-1), resulting in significant activation of proteins involved in phase II anti-oxidant response in HCT $116^{\text {TP53-/- }}$ and H1299 cells, which was reverted by NAC pretreatment (Figures 2c-e, Supplementary Figures S2A-C). This strongly implies that WA induces oxidative stress in cancer cells.

We reasoned that ROS induced by WA might promote TAp73 protein stabilization as NQO1, which expression depends on the oxidative status of the cell, was found to have a key role in ablating the Ub (ubiquitin)-independent degradation of p53 and TAp73. ${ }^{20}$ Indeed, pretreatment with NAC prevented the induction of TAp73 and PUMA proteins by WA (Figure 2e) and accordingly ablated the expression of TAp73 target genes PUMA and NOXA (Figure 2f). Furthermore, $1 \mu \mathrm{M}$ WA did not induce ROS in NHDF, which provides the explanation why normal cells are not affected by low dose of WA (Supplementary Figure S2D).

Thus WA promoted NRF2-related phase II anti-oxidant response as a consequence of induced oxidative stress. Next, our data indicate that oxidative stress induced by WA is critical for TAp73 stabilization and expression of its apoptotic targets PUMA and NOXA.

TAp73 is essential for potent killing of cancer cells lacking p53 upon WA treatment. As ROS is an important activator of TAp73, we strived to investigate how WA-induced ROS activates TAp73 in cancer cells and whether TAp73 contributes to cell killing facilitated by WA.

To explore the role of TAp73 in WA-mediated cell death, we used both transient and stable TAp73 knockdown cell lines for further studies (Supplementary Figure S3B). Silencing of TAp73 expression, using two different shRNAs, led to the significant protection from WA-induced growth inhibition (Figures 3a-d, Supplementary Figures S3C and D and S7A). To elucidate whether TAp73 contributes to potent activation of apoptotic effectors by WA, we performed western blotting analysis in shVector- and shTAp73expressing cells after WA exposure. We found that TAp73 depletion significantly reverted the induction of PARP cleavage, Bax and Bid accumulation by WA (Figures $3 e$ and f), which implies that TAp73 is required for the promotion of apoptotic program upon WA. Additionally, this was further confirmed by the less prominent accumulation of propidium iodide-stained cancer cells' knockdown for TAp73 and treated with WA (Figure $3 \mathrm{~g}$ ).

Phosphorylation of TAp73 by ROS-activated c-Jun N-terminal kinase (JNK) potentiates cell death triggered by WA. It has been described previously that genotoxic stress induced by cisplatin activates c-Abl kinase, which in turn phosphorylates TAp73 at Tyr-99 leading to protein stabilization and activation. ${ }^{50}$ Therefore, in most of our studies we applied cisplatin (CDDP) as a positive control. The MAPK cascade includes p38 MAPK and JNK/SAPK (stress-activated protein kinase), which are important signal transduction messengers that are relevant for the regulation of cell motility, transcription and apoptosis. p38 and JNK/ SAPK were shown to phosphorylate and activate TAp73 upon cellular stresses, ${ }^{51}$ which makes them candidate mediators of WA-induced oxidative stress to TAp73. Thus we found that WA promoted JNK and p38 kinases phosphorylation, which correlated with the phosphorylation of Tyr-99 in TAp73 (Figure 4a, Supplementary Figures S4A and B). TAp73-pTyr99 is a mark of DNA damage response and is executed by c-Abl kinase. ${ }^{52}$ As no DNA damage by $1 \mu \mathrm{M}$ WA was detected (Figure $1 \mathrm{~g}$ ), we speculate that the induction of pTyr99 might be a consequence of mitochondrial oxidative stress-mediated activation of DDR and is rather a downstream effect of the on-going apoptosis but not the DNA damage stress. ${ }^{53}$

The JNK kinase recognizes and phosphorylates serine and threonine residues, we thus performed phospho-specific IP for TAp73, which showed robust phosphorylation of $\mathrm{p} 73$ at multiple Ser and Thr residues 
a

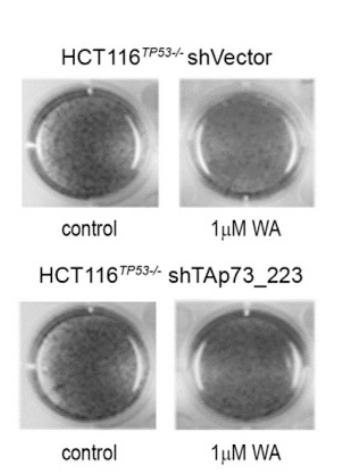

b

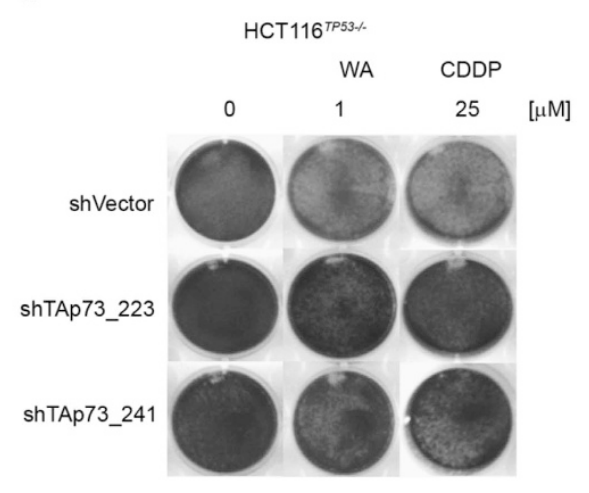

C

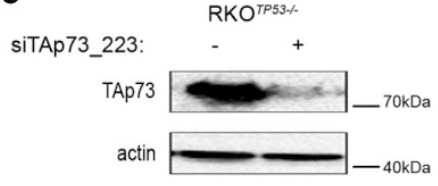

SiTAp73_223: $\quad-\quad+$

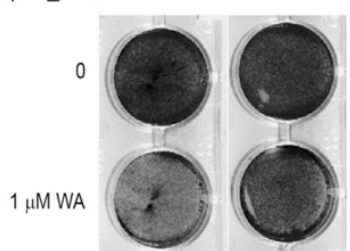

d

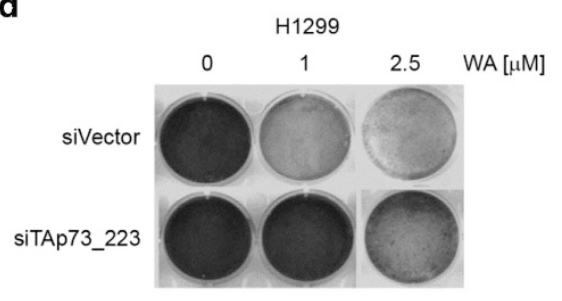

HCT116 ${ }^{\text {TP53 }}$

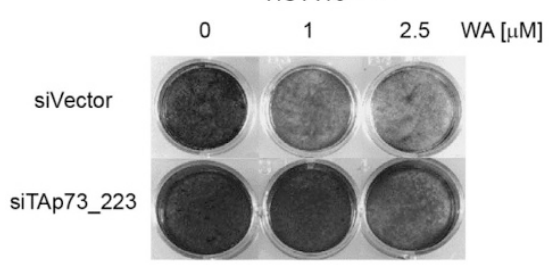

f

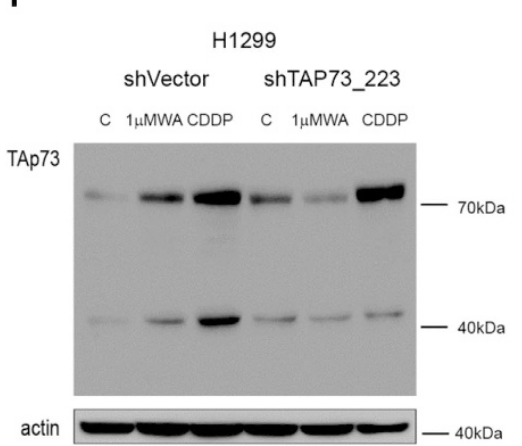

e

H1299

g
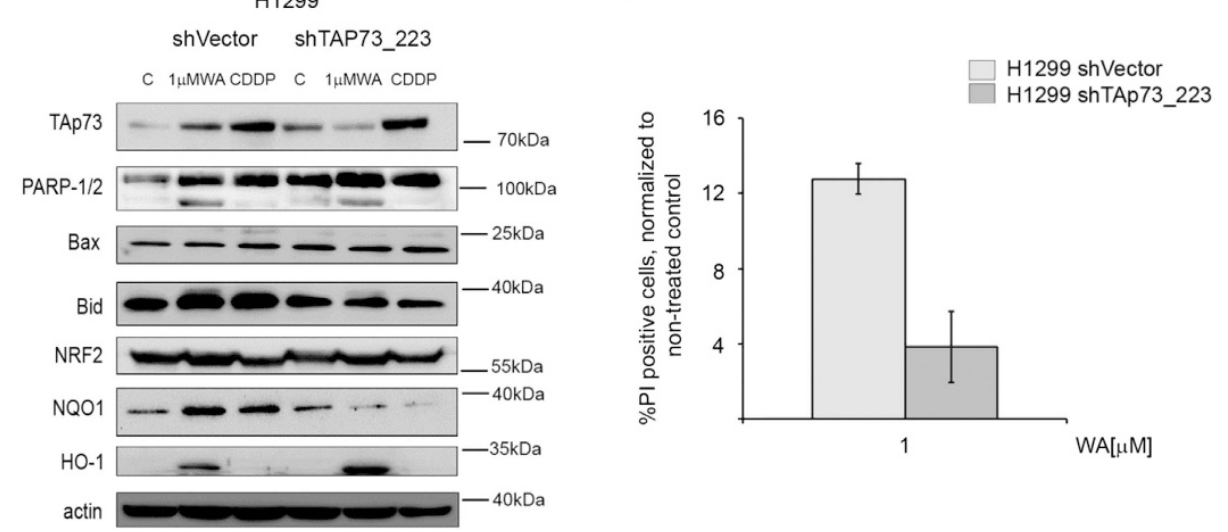

Figure 3 TAp73 directly contributes to cell killing by WA. (a) Soft agar assay performed in HCT116 ${ }^{\text {TP53-I }}$ - stably knockdown for TAp73 cells and shVector transduced counterpart, shows reduced potential of these cells to form colonies upon WA treatment only when TAp73 is expressed. This is representative data of 3 independent experiments. (b-d) Stable and transient silencing of TAp73 ablates anti-proliferative function of WA in cancer cells as revealed by clonogenic assays. (e) Depletion of TAp73 in HCT116 TP53-I- $^{-}$cells prevents induction of the pro-apoptotic PUMA by WA. The shRNA control and shTAp73 samples were analyzed on the same membrane and exposed at the same time. (f) Whole membrane representing the levels of TAp73 upon shRNA-mediated knockdown indicates ineffective induction of TAp73 after WA treatment. (g) Propidium iodide staining of viable HCT116 ${ }^{\text {TP53-I- }}$ shTAp73_223 shows reduced sensitivity to WA upon TAp73 depletion

upon WA treatment (Figure 4b). To confirm that JNK kinase is responsible for TAp73 phosphorylation, we pretreated the cells with JNK-specific inhibitor SP600125, which ablated the phosphorylation of threonine residues in TAp73
(Figure 4d). Interestingly, WN, an analog of WA and $\mathrm{PI}$ (Supplementary Figure S5A), induced TAp73 protein levels but did not promote the phosphorylation of TAp73 (Figure 4d, lanes 5 and 6). Additionally, JNK inhibitor not only 
a

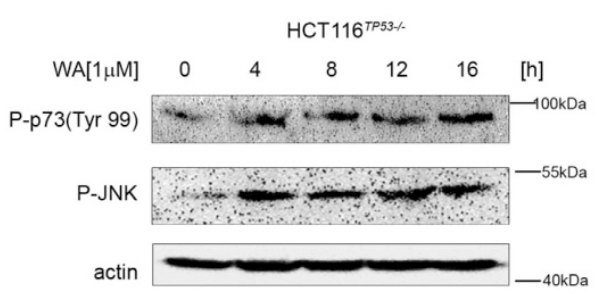

C
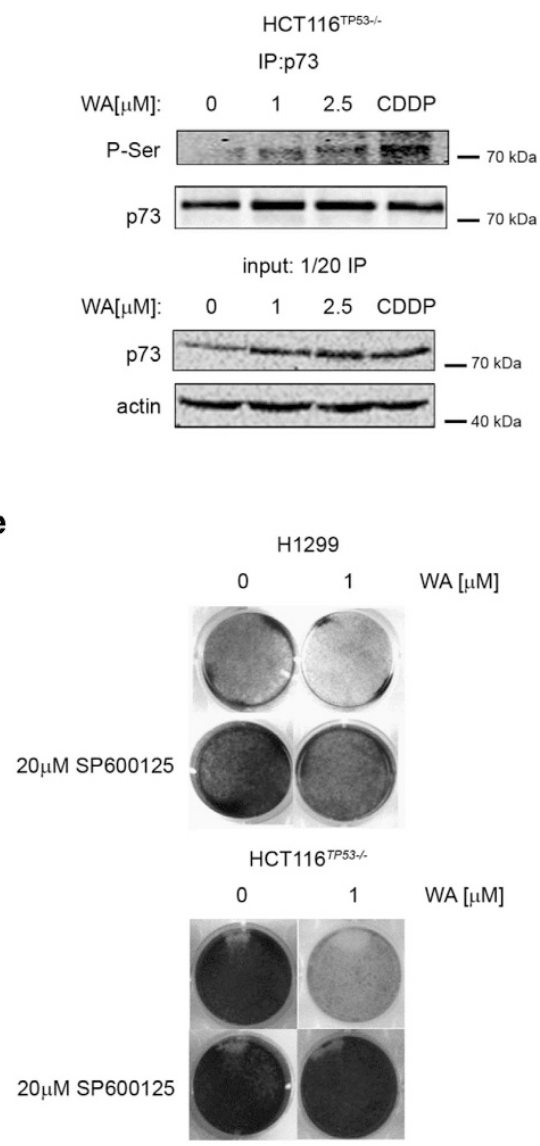

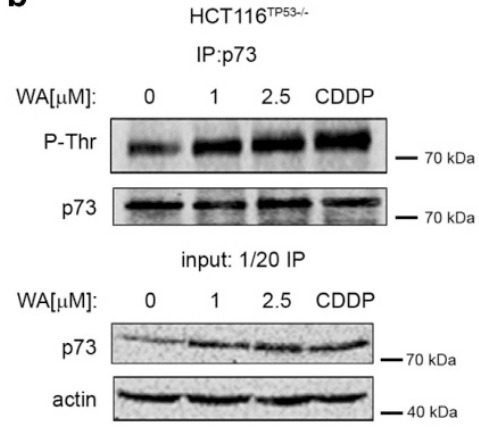

d

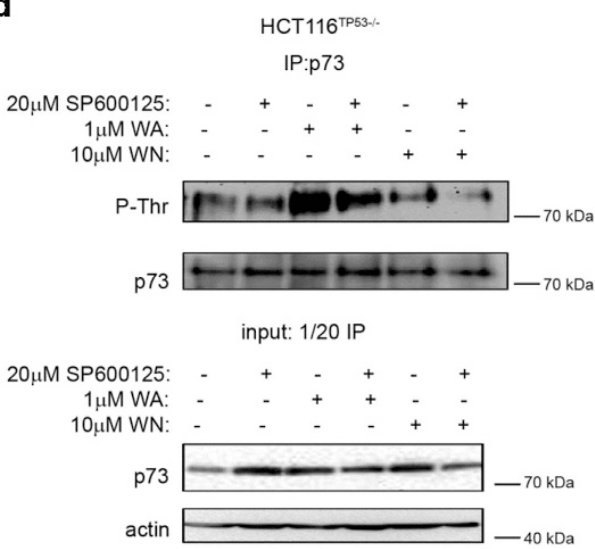

f

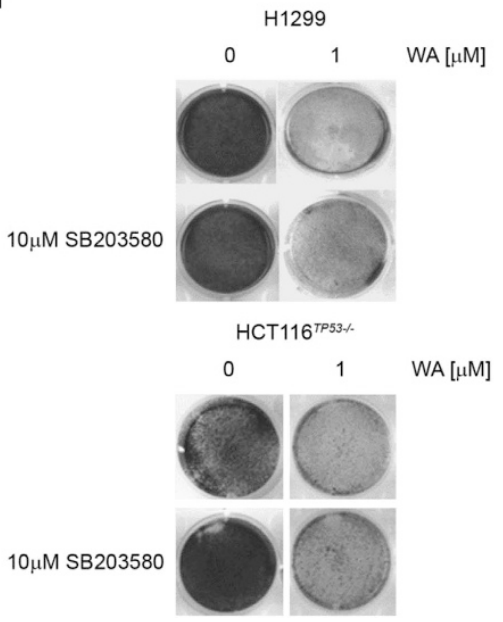

Figure 4 ROS-activated JNK kinase phosphorylates TAp73 and contributes to cell killing by WA. (a) WA triggers the phosphorylation of JNK kinase and TAp73. (b and c) WA promotes phosphorylation of threonine and serine residues in TAp73. (d) The SP600125 JNK inhibitor prevents phosphorylation of TAp73 on threonine residues by WA. (e) $20 \mu \mathrm{M}$ SP600125 significantly protects from WA-mediated growth inhibition. (f) SB203580, a p38 kinase inhibitor, does not protect H1299 and HCT116 ${ }^{\text {TP53 }-I-}$ cells from WA-mediated growth inhibition

impeded the phosphorylation of TAp73 but also prevented WA-induced growth inhibition (Figure 4e, Supplementary Figure S7B). In contrast, an inhibitor of p38 kinase (SB203580) did not prevent the growth inhibition triggered by WA (Figure 4f, Supplementary Figure S7C), thus our findings imply that JNK kinase is responsible for TAp73 phosphorylation and mediates the anti-proliferative activity of WA.
ROS-activated JNK stabilizes TAp73 protein levels via NRF2-NQ01 axis, which is synthetic lethal with the activation of TAp73 transcriptional activity. Next, we focused on elucidating the mechanism of TAp73 protein stabilization upon $1 \mu \mathrm{M}$ WA as evident in chase experiments (Figure 5a). In the present study, we have found that WA induced oxidative stress and activated the NRF2-related antioxidant response (Figures $2 \mathrm{a}$ and $\mathrm{c}$ ), which is prevented by 
a

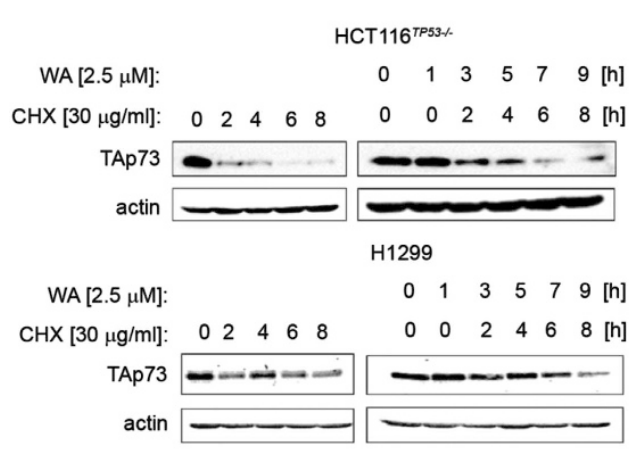

C

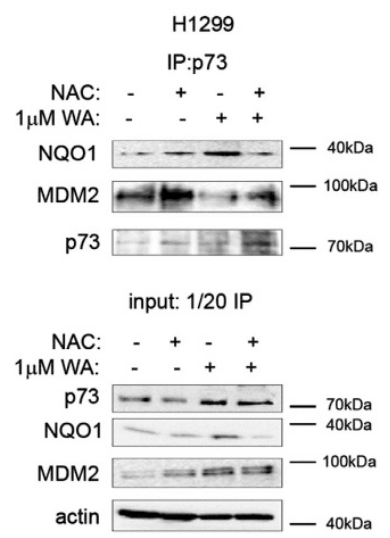

e

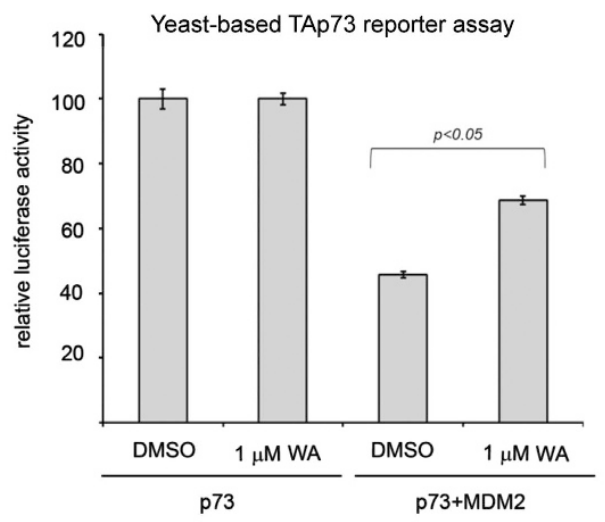

b

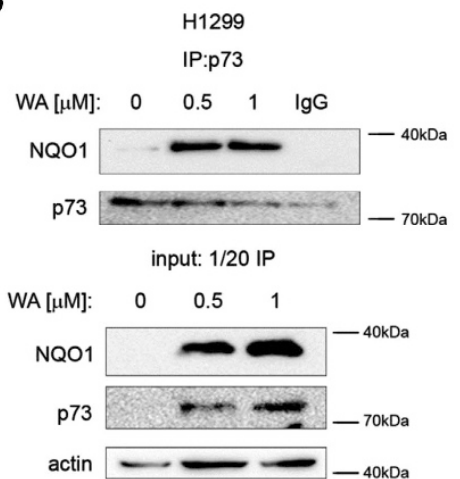

d

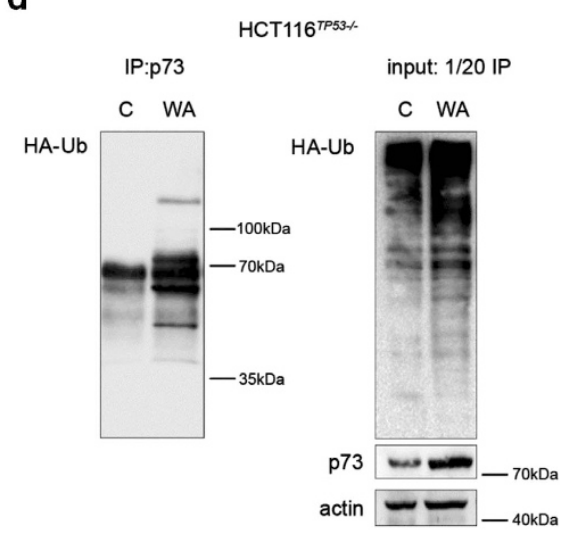

f

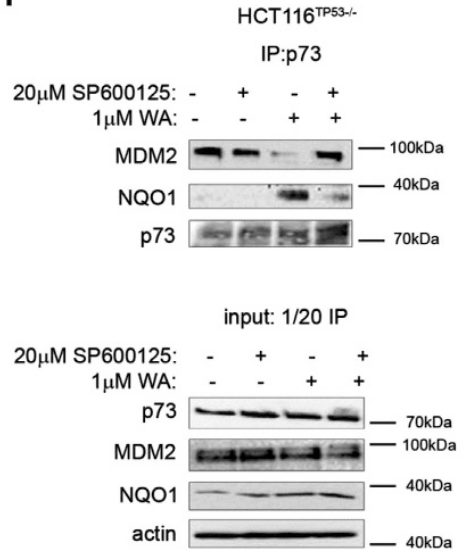

Figure 5 JNK stabilizes TAp73 by Nrf2-NQ01 axis, which is synthetic lethal with TAp73 phosphorylation. (a) TAp73 is stabilized in HCT116 TP53-/- and H1299 cells pretreated for $1 \mathrm{~h}$ with WA as shown by $\mathrm{CHX}$ chase experiment. (b) WA facilitates TAp73/NQ01 binding as revealed by co-immunoprecipitation experiment. (c) NAC prevents the binding of NQO1 to TAp73 promoted by WA and prevents WA-mediated inhibition of MDM2-p73 complex. (d) Accumulation of Ub-tagged TAp73 in WA-treated HCT116 ${ }^{\text {TP53-I- }}$ cells. (e) Co-expression of MDM2 inhibits TAp73-dependent transactivation in a yeast-based reporter assay, which is partially restored by WA $(n=4)$. (f) In the presence of JNK inhibitor, WA did not inhibit TAp73/MDM2 complex

NAC pretreatment. It has been reported previously that ROS-activated JNK phosphorylates NRF2 and promotes its transcriptional activity. ${ }^{54,55}$ This is in agreement with our observation that $1 \mu \mathrm{M}$ WA stabilizes NRF2 protein as evidenced by the elevated mRNA and protein levels of NF-E2-related transcription factor (NRF2) target genes NQO1 and HMOX-1 (Figures 2c and d). NQO1 is a 20S proteasomal gatekeeper, which under oxidative stress directly binds to $\mathrm{p} 53$ and TAp73a and rescues them from degradation by inhibition of $20 \mathrm{~S}$ proteolytic activity. ${ }^{20}$ Our immunoprecipitation analysis revealed that WA promoted NQO1 binding to TAp73 (Figure 5b). This binding was at least partially dependent on ROS, as NAC pretreatment significantly reduced the binding of NQO1 to TAp73 upon WA treatment (Figure 5c). Further, NQO1-TAp73 binding promoted accumulation of Ub-tagged TAp73 (Figure 5d), a mark 
of inhibited proteasomes. As WA directly inhibits catalytic activity of $20 S$ proteasome, ${ }^{26}$ we concluded that both ROSNrf2-NQO1 and direct inhibition of $20 \mathrm{~S}$ contributes to potent TAp73 stabilization by WA.

Phosphorylation of p53 at serine 15 and 20 is a mark of p53 activation leading to ablation of p53/MDM2 complex, p53 protein stabilization and promotion of the binding of p53 coactivators. ${ }^{56}$ As TAp73 transcriptional activity is abrogated by overexpressed MDM2 and MDMX proteins, which directly bind to and inhibit transactivation domain of TAp73, ${ }^{52}$ we investigated whether WA treatment could ablate the interaction between TAp73 and MDM2. To address this, we first used a defined yeast-based reporter system, which detects TAp73 transcriptional activity by using as a readout the functionality of a TAp73-dependent luciferase reporter. As MDM2 does not degrade TAp73, thus in this setup the inhibition of reporter is related to the direct binding of MDM2 to TAp73. ${ }^{57}$ Cotransfection of TAp73 with MDM2 inhibited the TAp73 reporter, which was counteracted by WA treatment (Figure 5e). In addition, WA efficiently disrupted TAp73/MDM2 complex in HCT $116^{\text {TP53-I- }}$ cells (Figure 5f), which was dependent on ROS as manifested by the lack of inhibition in NAC pretreated samples (Figure 5c).

To elucidate whether the TAp73 phosphorylation is relevant for the ablation of TAp73/MDM2 complex, we pretreated the cells with JNK inhibitor prior to WA treatment. We found that inhibition of JNK kinase prevents the release of TAp73 from the inhibitory complex with MDM2 protein (Figure 5f).

Hence, we concluded that WA-induced oxidative stress promotes JNK-dependent TAp73 phosphorylation, which leads to the inhibition of TAp73/MDM2 complex and TAp73 activation.

Next, we strived to investigate whether other inhibitors of $20 S$ proteasome work in the way similar to WA. To address this, we used its structural analog - WN (Supplementary Figure S5A), which has been reported to inhibit $20 S$ proteasome catalytic subunit. ${ }^{24}$ Surprisingly, we found that WN induced TAp73 at the protein levels but did not promote TAp73 phosphorylation (Figure 4d). Additionally, WN only slightly inhibited proliferation of cancer cells at a high dose (Supplementary Figure S5B) and did not promote apoptotic phenotype in treated cancer cells H1299 and HCT $116^{\text {TP53-I- }}$ (Supplementary Figure S5D). We found that this is attributed to the fact that WN does not induce efficient oxidative stress in cancer cells at the concentrations tested (Supplementary Figure S4D).

Taken together, our data suggests that WA by inducing ROS activates JNK-dependent phosphorylation and consequent activation of TAp73 by targeting TAp73/MDM2 interactions. Concurrently, WA promotes NQO1-driven stabilization of TAp73, which confers synthetic lethality with JNK-related TAp73 activation in cancer cells.

\section{Discussion}

TAp73 is a tumor suppressor that together with TAp63 can recognize many of p53 target genes involved in cell cycle regulation and apoptosis. ${ }^{50,58,59}$ In addition, TAp73 has also unique roles in neuronal stem cells' differentiation ${ }^{60}$ and metabolism. ${ }^{37}$ However, the critical function of TAp73 in tumor suppression is still not fully resolved due to the tissue-specific response deriving from the existence of the p73 isoforms often possessing opposing functions to each other. ${ }^{33}$ This makes the estimation of TAp73 clinical relevance much more complex when compared with p53. ${ }^{33}$ Unlike TP53, TP73 gene is rarely mutated, and the functionality of TAp73 is mainly ablated by inhibitory interactions with $\triangle N p 73, M D M 2, M D M X$, iASPP or mutant $\mathrm{p53} .^{52}$ Thus, targeting protein-protein interactions or modulating pathways promoting TAp73 posttranslational modifications serve as the promising approach for treatment of tumors where p53 is lost or mutated. Several small molecules have recently been reported to positively affect TAp73 apoptotic pathway, including RETRA, ${ }^{44}$ Nutlin- $3^{42}$ and rapamycin; ${ }^{61}$ however, their widespread application to promote p73-dependent tumor regression is still limited. ${ }^{34}$

Eliciting the mechanism underlying cell death evoked by pharmacologically activated TAp73 is of great relevance for the future effective utilization of TAp73 functionality to treat tumors. ${ }^{40}$

In the present work, we report that non-genotoxic oxidative stress synergizes with the inhibition of 205 proteasome to effectively trigger TAp73-related apoptosis in cancer cells using single agent - small-molecule WA. We have shown that WA triggers oxidative stress and that the ROS-activated JNK kinase promotes potent induction of TAp73 protein levels. Further, we elucidated the mechanisms downstream of JNK, which has a crucial role in TAp73 activation in cancer cells where TP53 gene is lost or depleted.

Previously, Selivanova and colleaques have shown that the inhibition of thioredoxin reducatase (TrxR), important anti-oxidant enzyme often upregulated in cancers, is synthetic lethal with p53 activation and that ROS-activated JNK mediates this effect. ${ }^{46}$ Here, using two small-molecule inhibitors of the $20 \mathrm{~S}$ proteasome, we demonstrated that the activation of JNK and inhibition of $20 \mathrm{~S}$ proteasome confers synthetic lethality in cancer cells.

The approval of bortezomib for the treatment of multiple myeloma $^{21}$ and the ongoing clinical trials with the new, potent proteasomal inhibitor AP15 recently identified in a cell-based screen, ${ }^{21,62}$ provide solid rationale for targeting proteasomes for effective anti-cancer treatment. p53 and TAp73 can be stabilized upon IR-mediated ROS in a way independent from ubiquitin, by direct binding to NQO1, ${ }^{20}$ which facilitates the inhibition of $20 \mathrm{~S}$ proteasome and rescues p53 and TAp73 from degradation. However, the upstream mechanisms leading to the stabilization of TAp73 by ROS insults were not previously described. Our data imply that WA-induced ROS activates JNK kinase, which promotes stabilization of NRF2 transcription factor. This triggers expression of phase II anti-oxidant effectors, including HO-1 and NQO1. Next, we showed that stabilized NQO1 binds to TAp73a and promotes accumulation of Ubtagged TAp73 (Figures $5 \mathrm{c}$ and d). This is in agreement with the study by Asher et al., ${ }^{20}$ who demonstrated that NQO1 is critical for Ub-independent turnover of p53 and TAp73. By employing small-molecule WN, an analog of WA and effective inhibitor of $20 \mathrm{~S}$ proteasome, ${ }^{25}$ we discovered a novel mechanism leading to synthetic lethal effect in cancer cells, converging on ROS-dependent, JNK-driven phosphorylation and activation of TAp73 and simultaneous 


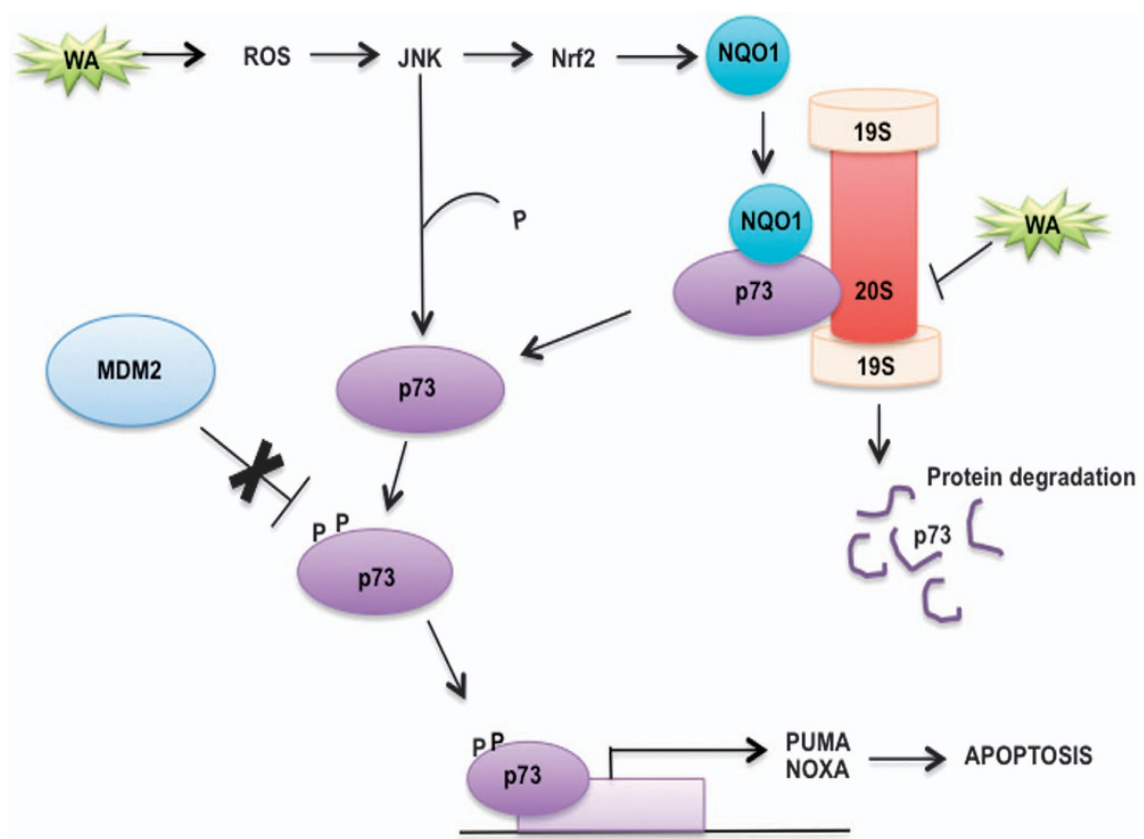

Figure 6 Model showing synergistic mechanism of TAp73 activation by WA via JNK, which is synthetic lethal with inhibition of TAp73 proteasomal degradation. ROS insult activates JNK, which triggers NRF2-dependent expression of NQ01. NQO1 binds to TAp73 and prevents its degradation by 20S proteasome, which leads to TAp73 protein stabilization. JNK also puts phosphorylation marks to promote TAp73 transcriptional activity by ablating TAp73/MDM2 complex. This leads to the activation of proapoptotic PUMA and NOXA, which is indispensable for efficient tumor killing. Thus dual targeting of TAp73/MDM2 and 20S proteasome leads to potent apoptosis in cancer cells deficient for p53

TAp73 protein stabilization. Based on our data, we propose a model showing how ROS-activated JNK stabilizes TAp73 through activation of NRF2 and its downstream target NQO1, which together with the release of TAp73 from MDM2 leads to activation of TAp73-dependent apoptotic response (Figure 6). We speculate that the activation of the proapoptotic PUMA might further lead to the amplification of ROS insult in cancer cells and provide additional fueling of the activating signals to JNK in a way similar to how we have recently described for the pharmacologically activated p53. ${ }^{46}$

Elucidating the mechanisms by which pharmacologically activated TAp73 promotes apoptosis in cancer cells but not in normal cells is of great importance for the effective therapeutic targeting of TAp73 in the clinical setting. It has been shown that restoration of p53 functionality in advanced tumors but not in early lesions leads to potent cancer regression. ${ }^{56}$ Our present findings imply that WA by promoting ROS provokes accumulation of NQO1, which inhibits 205 proteasome and stabilizes TAp73. Our finding is novel as it describes previously unrecognized pathway of efficient activation of TAp73. We speculate that the same mechanism triggered by a smallmolecule WA can lead to the activation of all p53 family members, including $\mathrm{p53}$, and this will be the subject of future studies. Similar mechanism regarding the protein maintenance by activated NQO1 was reported for the translation initiation factor 4GI (elF4GI); however, in that study, the link to how the oxidative stress might contribute to this process was not recognized. ${ }^{63}$

Thus our novel findings describing synthetic lethality between inhibition of proteasome system and ROS- dependent activation of MAPK JNK kinase for potent cancer cell death constitutes an attractive anti-cancer approach, which might serve as an important rationale for the development of effective treatment of ROS-compromised tumors by small molecules like WA.

\section{Materials and Methods}

Cell lines and chemicals. Tumor cell lines HCT116 ${ }^{\text {TP53-I- }}$ and RKO ${ }^{T P 53-1-}$ were provided by B Vogelstein, John Hopkins University, USA. HCT116 ${ }^{\text {TP53-I- }}$, RKO ${ }^{\text {TP53-l- }}$ H1299 and NHDFs were maintained in Iscove's Modified Dulbecco's Medium (IMDM) supplemented with 10\% fetal bovine serum (FBS), L-glutamine $(2 \mathrm{mM})$ and penicillin/streptomycin (10 units/ml). All cell lines were cultured at $37^{\circ} \mathrm{C}$ in a humidified incubator with $5 \% \mathrm{CO}_{2}$.

WA was purchased from Sigma-Aldrich (Santa Cruz, St. Louis, MO, USA) and dissolved in $100 \%$ DMSO to $1 \mathrm{mM}$ concentration. Cisplatin (CDDP) was purchased from Sigma-Aldrich and diluted in $0.9 \% \mathrm{NaCl}$ to $25 \mathrm{mM}$ solution and added to the culture medium of $25 \mu \mathrm{M}$ final concentration. NAC (Sigma-Aldrich) was dissolved in $\mathrm{dH}_{2} \mathrm{O}$ to a $0.5 \mathrm{M}$ solution and further added to cell culture medium to a $5 \mathrm{mM}$ concentration. SP600125 JNK inhibitor was purchased from Sigma-Aldrich, dissolved in $100 \%$ DMSO and used at $20-30 \mu \mathrm{M}$ concentration.

zVAD-FMK was purchased from Sigma-Aldrich and used at $10 \mu \mathrm{M}$ concentration.

siRNA and plasmids. For TAp73 knockdown, following siRNAs were used: TAp73_318 sense: 5'-AGGGCAUGACUACAUCUGU-3'; antisense: 5'-ACAGAUG UAGUCAUGCCCU-3' and TAp73_223 sense: 5'-ACCAGACAGCACCUACUUC-3'; antisense: 5'-GAAGUAGGUGCUGUCUGGU-3'. Transfection with siRNA (10 nM) was performed with HiPerfect Qiagene (Qiagen, Germantown, MD, USA) reagent for $24 \mathrm{~h}$ (for WB analysis) or $48 \mathrm{~h}$ (for colony-formation assay) following the manufacturer's instruction.

Plasmid encoding for TAp73 $\alpha$ isoform was a kind gift from Dr. Matthias Dobbelstein. Transfection was performed with Lipofectamine 2000 Reagent (Life Technologies, Thermo Fisher Scientific, Carlsbad, CA, USA), according to the manufacturer's protocol. 
Generation of stable TAp73 knockdown cell lines. To establish stable TAp73 knockdown HCT116 ${ }^{\text {TP53-I- }}$ and H1299 cell lines, we used two different shRNA constructs directed against human TAp73. The shRNA oligos were cloned into pLKO.1-puro vector (Addgene, Cambridge, MA, USA; plasmid 8453) as previously described by Stewart et al.; $; 4$ oligo sequences were $5^{\prime}$-CCGGAC CAGACAGCACCTACTTCTTCAAGAGAGAAGTAGGTGC TGTCTGGTTTTTTG-3' and 5'-CCGGGAACGGATTCCAGCATGGATTCAAGAGATCCA TGCTGGAATCCGT TCTTTTTG-3'. Packaging and envelope constructs pCMV $\Delta 8.2$ and pMD.G-VSV-G were used for lentivirus generation via transfection into HEK293T cells as previously described in Szulc et al. ${ }^{65}$ In all, 16000 cells/well were seeded in 96-well plates and transduced with either control lentivirus or lentivirus containing TAp73 shRNA and selected by growing in puromycin $(1 \mu \mathrm{g} / \mathrm{ml})$ for $3-4$ weeks. $^{64,65}$

Anchorage-independent growth by soft agar assay. $1.2 \%$ Lowmelting temperature agarose (Sea plaque agarose, Lonza Group Ltd, Basel, Switzerland) was melted and mixed with an equal volume of warm $2 \times$ Dulbecco's Modified Eagle's Medium (DMEM) (containing 20\% FBS, 4 mM L-glutamine, 2\% penicillin/streptomycin) to prepare $0.6 \%$ base agarose. A total of $600 \mu \mathrm{l}$ of this mixture was added per 12-well and solidified for $30 \mathrm{~min}$ at RT. The top agarose $(0.4 \%$ agarose) was prepared mixing $0.8 \%$ low-melting temperature agarose with $2 \times$ DMEM-containing cells. Ten thousand cells per 12-well was added onto the base agarose and solidified for $30 \mathrm{~min}$ at RT. In all, $500 \mu$ medium containing WA was added onto top agarose. After 1 week of culture, colonies were stained with MTT solution $(10 \mathrm{mg} / \mathrm{ml})$ and scanned.

Primary MEFs. $\mathrm{TAp}^{+/ /+}$and $\mathrm{TAp} 73^{-1-}$ primary MEFs were isolated from E13.5 embryos and cultured in DMEM supplemented with 10\% FBS, $2 \mathrm{mM}$ L-glutamine and $55 \mu \mathrm{M} \mathrm{b}$-mercaptoethanol. All animal experiments were conducted in accordance with the guidelines of Karolinska Institute and approved by the Stockholm's North Ethical Committee of Animal Research.

Cell viability assay. For viability studies, cells were plated at a concentration of $5 \times 10^{3}$ cells/well, and WA was added in IMDM medium at increasing concentrations, and $25 \mu \mathrm{M}$ CDDP was used as a positive control. After $48 \mathrm{~h}$, absorbance of WST-1 (2-(4-iodophenyl)-3-(4-nitrophenyl)-5-(2,4-disulfophenyl)-2Htetrazolium, monosodium salt, Roche Diagnostics, Penzberg, Germany) dye was measured in a Perkin-Elmer (Waltham, MA, USA) microplate reader, and cell viability was calculated.

Clonogenic survival assay. Cells were plated at concentrations of $1.5 \times 10^{5}$ per well or $2 \times 10^{5}$ for stable TAp73 knockdown cell lines, and WA was added in increasing concentrations. After $48 \mathrm{~h}$, medium was removed, and cells were washed with PBS, followed by $70 \%$ ethanol and stained with $0.5 \%$ crystal violet solution in PBS. Densitometric analysis of relative well density was performed using the ImageJ software (Bethesda, MD, USA).

Quantitative PCR. mRNA was isolated and reverse transcribed to cDNA according to the manufacturer's instructions (Bio-Rad, Sundbyberg, Sweden). For qPCR, the following concentrations were used: $150 \mathrm{nM} ; 10 \mathrm{ng} \mathrm{CDNA} ; 7.5 \mu \mathrm{l}$ $2 \times$ master mix (Bio-Rad); water to a total of $15 \mu$ l; primers used: NOXA (PMAIP1) forward 5'-AAGTGCAAGTAGCTGGAAG-3', reverse: 5'-TGTCTCCAAATCTCCT GAGT-3', PUMA forward: 5'-CTCAACGCACAGTACGAG-3' and reverse: $5^{\prime}$-GTCC CATGAGATTGTACAG-3', NFLE2E forward: 5'-TCCCAGCAGGACATGGATTT-3 and reverse: 5'-GCTCATACTCTTTCCGTCGC-3', HMOX-1 forward: 5'-TTCAC CTTCCCCAACATTGC-3' and reverse: 5'-TATCACCCTCTGCCTGACTG-3', BCl-2 forward: 5'-GCCTTCTTTGAGTTCGGT-3' and reverse: 5'-AGTTCCACAAAGGC ATCC-3', Bid forward: 5'-GTGAGGTCAACAACGGTTCC-3' and reverse: $5^{\prime}$-TGCC TCTATTCTTCCCAAGC-3', Bim forward: 5'-TGGCAAAGCAACCTTCTGATG-3' and reverse: 5'-GCAGGCTGCAATTGTCTACCT-3', $\triangle$ Np73 forward: 5'-CAAACGG CCCGCATGTTCCC-3' and reverse: 5'-TTGAACTGGGCCGTGGCGAG-3', and TAp73 forward: 5'-GGGAATAATGAGGTGGTGGG-3' and reverse: 5'-AGATTG AACTGGGCCATGAC-3'

ROS measurement. ROS were measured as described previously. ${ }^{66}$ Briefly, cells were treated with WA or pretreated with $5 \mathrm{mM} \mathrm{NAC}$ or $100 \mu \mathrm{M} \mathrm{H}_{2} \mathrm{O}_{2}$ for $6 \mathrm{~h}$ and stained with $10 \mu \mathrm{M}$ DCF-DA (Sigma-Aldrich) in serum-free IMDM for $45 \mathrm{~min}$. After three washing steps with PBS, cells were trypsynized and suspended in PBS. Samples were analyzed by flow cytometry, acquiring 10000 cells per sample. Data analysis was performed with the CELLQuest software (CELLQuest, Franklin Lakes, NJ, USA).

Cell cycle analysis by flow cytometry. Cells were stained with propidium iodide solution (Sigma-Aldrich). Samples were washed, suspended in PBS and analyzed by flow cytometry, covering acquisition of 10000 cells per sample. Data analysis was performed with the CELLQuest software.

Co-immunoprecipitation and western blottig analysis. Cells for both whole-cell lysates and immunoprecipitates were solubilized in lysis buffer: $25 \mathrm{mM}$ Tris HCl, pH 8.0, $150 \mathrm{mM} \mathrm{NaCl}$, and 1\% Nonidet P-40 (0.5\% for co-IP). For co-IP, $1 \mathrm{mg}$ protein was precleared with $10 \mu \mathrm{l}$ Dynabeads Protein A (Invitrogen, Carlsbad, CA, USA) coupled with normal rabbit lgG. Precleared lysates were then immunoprecipitated for $16 \mathrm{~h}$ with $1.5 \mu \mathrm{g}$ of $\alpha$-TAp73 rabbit polyclonal antibody (Bethyl Laboratories, Montgomery, TX, USA). Immunocomplexes were incubated with $20 \mu \mathrm{l}$ of Dynabeads Protein A for $5 \mathrm{~h}$ at $4{ }^{\circ} \mathrm{C}$ to form complexes. The immunoprecipitates were washed two times with $300 \mu \mathrm{l}$ of PBS and two times with $200 \mu \mathrm{l}$ of lysis buffer, resuspended in $15 \mu \mathrm{l}$ of lysis buffer and $10 \mu \mathrm{l}$ of $6 \times$ Laemmlibuffer and boiled prior to western blotting analysis. The antibodies used for detection were: anti-p73 antibodies (Bethyl Laboratories), anti-MDM2 (Santa Cruz Biotechnology, Dallas, TX, USA), anti-NQ01 (Santa Cruz Biotechnology), anti-HA (Santa Cruz Biotechnology) and anti-phospho-threonine (Cell Signaling, Danvers, MA, USA).

Western blotting was performed according to the standard protocol. Briefly, $100 \mu \mathrm{g}$ of total cell lysate $(200 \mu \mathrm{g}$ for phosphorylated protein detection) was subjected to electrophoresis, and the following antibodies were used to detect proteins: anti-TAp73 (Bethyl Laboratories), anti-BAX (Santa Cruz Biotechnology) anti-PUMA (Cell Signaling), anti-HO (Santa Cruz Biotechnology), anti-NRF2 (Santa Cruz Biotechnology), anti-Pp73(Tyr99), anti-pJNK (Cell Signaling) anti-pp38 (Cell Signaling), anti-Bid (Santa Cruz Biotechnology), and anti-actin (Sigma).

Cycloheximide (CHX) protein stability assay. For $\mathrm{CHX}$ chase experiment, H1299 and HCT116 ${ }^{\text {TP53-I- }}$ cells were pretreated for $1 \mathrm{~h}$ with WA. In all, $30 \mu \mathrm{g} / \mathrm{ml} \mathrm{CHX}$ was then added to block protein translation. Cell pellets were collected, solubilized in lysis buffer and analyzed by western blotting as previously described.

Caspase activation assay. Detection of caspases was measured after $16 \mathrm{~h}$ treatment with WA with the use of FLICA (Fluorescent-Labeled Inhibitor of Caspases) apoptosis and caspase detection kit (ImmunoChemistry Technologies, Bloomington, MN, USA). In brief, cells were collected, washed with PBS and stained according to the manufacturer's protocol. Caspase activity was measured by flow cytometry, and collected results were analyzed with CELLQuest software.

Yeast-based reporter system. The TAp73-dependent yeast reporter strain yLFM-PUMA containing the luciferase cDNA cloned at the ADE2 locus and expressed under the control of a promoter element derived from the human PUMA gene was transfected with pTSG-p73 and pRB-MDM2 (generously provided by Dr. R Brachmann, University of California, Irvine, CA, USA) and selected on double drop-out media for TRP1 and HIS3. Expression of p73 was controlled by the addition of galactose in the culture medium, while MDM2 expression was constitutive. Luciferase activity was measured $16 \mathrm{~h}$ after the shift of double transformant cells to galactose-containing selective media and the addition of WA or DMSO. Presented are average relative light units and the S.E. obtained from three independent experiments each containing four biological repeats. In all cases $t$ Student test was performed to assess statistically relevant differences.

Comet assay. DNA strand breaks were detected by comet assay as previously described ${ }^{67}$ Briefly, following treatment, cells were mixed with $100 \mu \mathrm{l}$ of $1 \%$ low melting agarose (Prona, Reducta LM, Poland), and $75 \mu \mathrm{l}$ of this cell-agarose mixture was spread on microscopic slides precoated with $1 \%$ agarose. A third layer of $0.5 \%$ low-melting agarose $(75 \mu \mathrm{l})$ was applied over the layer of agarose with the cell suspension. Slides were incubated for $1 \mathrm{~h}$ in a lysis solution. The microscopic slides were immersed in an alkaline buffer ( $300 \mathrm{mM} \mathrm{NaOH}, 1 \mathrm{mM}$ disodium EDTA) and subjected to electrophoresis at $1 \mathrm{~V} / \mathrm{cm}$ for $30 \mathrm{~min}$. Stained cells were analyzed under a fluorescence microscope (Nikon, Tokyo, Japan PCM-2000) using the Cometscore software (Tri Tek Corp., Sumerduck, VA, USA). Images of 20 cells from 
three slides were analyzed. Densities were measured for each image in two areas: the whole cellular DNA and the area containing only the comet head. Results are presented as tail moment, which is the percentage of DNA in the comet tail multiplied by the tail length.

\section{Conflict of Interest}

The authors declare no conflict of interest.

Acknowledgements. This study was supported by the Karolinska Institutet/ Stockholm County Council (KI/SLL) (ACT! Theme Center) grant, IUVENTUS PLUS 0635/IP1/2011/71 Polish Ministry of Science, KI fonder (to JZP), EU Human Capital Program (to AS), ETIUDA1 grant from NSC no. 2013/08/T/NZ3/00714 Human Capital Operational Programme, Action 4.1.1 (to AS). JZ-P would like to acknowledge Young Investigator Award from Polish Ministry of Science. Special thanks are addressed to Professor G Selivanova from KI for valuable comments, scientific support and suggestions to the study, to Prof $\mathrm{K}$ Bielawski from Laboratory of Molecular Diagnostics from Intercollegiate Faculty of Biotechnology, UG Poland for scientific support and to all our colleagues who shared reagents and cell lines with us.

1. Trachootham D, Alexandre J, Huang P. Targeting cancer cells by ROS-mediated mechanisms: a radical therapeutic approach? Nat Rev Drug Discov 2009; 8: 579-591.

2. Comoglio PM, Giordano S, Trusolino L. Drug development of MET inhibitors: targeting oncogene addiction and expedience. Nat Rev Drug Discov 2008; 7: 504-516.

3. Frantz S. The trouble with making combination drugs. Nat Rev Drug Discov 2006; 5 : 881-882.

4. Ranjan P, Anathy V, Burch PM, Weirather K, Lambeth JD, Heintz NH. Redox-dependent expression of cyclin D1 and cell proliferation by Nox1 in mouse lung epithelial cells. Antioxid Redox Signal 2006; 8: 1447-1459.

5. Shi X, Zhang Y, Zheng J, Pan J. Reactive oxygen species in cancer stem cells. Antioxid Redox Signal 2012; 16: 1215-1228.

6. Boonstra J, Post JA. Molecular events associated with reactive oxygen species and cell cycle progression in mammalian cells. Gene 2004; 337: 1-13.

7. Dickinson $\mathrm{BC}$, Chang $\mathrm{CJ}$. Chemistry and biology of reactive oxygen species in signaling or stress responses. Nat Chem Biol 2011; 7: 504-511.

8. Finkel T. Signal transduction by reactive oxygen species. J Cell Biol 2011; 194: 7-15

9. Ma Q. Advances in mechanisms of anti-oxidation. Discov Med 2014; 17: 121-130.

10. Rotblat B, Melino G, Knight RA. NRF2 and p53: Januses in cancer? Oncotarget 2012; 3 : 1272-1283.

11. Gorrini C, Harris IS, Mak TW. Modulation of oxidative stress as an anticancer strategy. Nat Rev Drug Discov 2013; 12: 931-947.

12. Bossy-Wetzel E, Talantova MV, Lee WD, Schölzke MN, Harrop A, Mathews E et al. Crosstalk between nitric oxide and zinc pathways to neuronal cell death involving mitochondrial dysfunction and p38-activated K+ channels. Neuron 2004; 41: 351-365.

13. Colavitti R, Finkel T. Reactive oxygen species as mediators of cellular senescence. IUBMB Life 2005; 57: 277-281

14. Aiken CT, Kaake RM, Wang X, Huang L. Oxidative stress-mediated regulation of proteasome complexes. Mol Cell Proteomics 2011; 10(R110): 006924.

15. Multhaup G, Ruppert T, Schlicksupp A, Hesse L, Beher D, Masters CL et al. Reactive oxygen species and Alzheimer's disease. Biochem Pharmacol 1997; 54: 533-539.

16. Brown NS, Bicknell R. Hypoxia and oxidative stress in breast cancer. Oxidative stress: its effects on the growth, metastatic potential and response to therapy of breast cancer. Breast Cancer Res 2001; 3: 323-327.

17. Goldberg AL. Protein degradation and protection against misfolded or damaged proteins. Nature 2003; 426: 895-899.

18. Ding Q, Dimayuga E, Martin S, Bruce-Keller AJ, Nukala V, Cuervo AM et al. Characterization of chronic low-level proteasome inhibition on neural homeostasis. J Neurochem 2003; 86: 489-497.

19. Seifert U, Bialy LP, Ebstein F, Bech-Otschir D, Voigt A, Schröter F et al. Immunoproteasomes preserve protein homeostasis upon interferon-induced oxidative stress. Cell 2010; 142: 613-624.

20. Asher G, Tsvetkov P, Kahana C, Shaul Y. A mechanism of ubiquitin-independent proteasomal degradation of the tumor suppressors p53 and p73. Genes Dev 2005; 19 : 316-321.

21. D'Arcy $P$, Brnjic S, Olofsson MH, Fryknäs M, Lindsten $\mathrm{K}$, De Cesare $\mathrm{M}$ et al. Inhibition of proteasome deubiquitinating activity as a new cancer therapy. Nat Med 2011; 17: 1636-1640.

22. Vyas AR, Singh SV. Molecular targets and mechanisms of cancer prevention and treatment by withaferin $\mathrm{A}$, a naturally occurring steroidal lactone. AAPS J 2014; 16: 1-10.

23. Mohan R, Hammers HJ, Bargagna-Mohan P, Zhan XH, Herbstritt CJ, Ruiz A et al. Withaferin $\mathrm{A}$ is a potent inhibitor of angiogenesis. Angiogenesis 2004; 7: 115-122.
24. Yang $\mathrm{H}$, Shi $\mathrm{G}$, Dou QP. The tumor proteasome is a primary target for the natural anticancer compound Withaferin A isolated from "Indian winter cherry". Mol Pharmacol 2007; 71: 426-437.

25. Khan S, Rammeloo AW, Heikkila JJ. Withaferin A induces proteasome inhibition, endoplasmic reticulum stress, the heat shock response and acquisition of thermotolerance. PLoS One 2012; 7: e50547.

26. Khedgikar V, Kushwaha P, Gautam J, Verma A, Changkija B, Kumar A et al. Withaferin A: a proteasomal inhibitor promotes healing after injury and exerts anabolic effect on osteoporotic bone. Cell Death Dis 2013; 4: e778.

27. Oh JH, Kwon TK. Withaferin A inhibits tumor necrosis factor-alpha-induced expression of cell adhesion molecules by inactivation of Akt and NF-kappaB in human pulmonary epithelial cells. Int Immunopharmacol 2009; 9: 614-619.

28. Bargagna-Mohan P, Ravindranath PP, Mohan R. Small molecule anti-angiogenic probes of the ubiquitin proteasome pathway: potential application to choroidal neovascularization. Invest Ophthalmol Vis Sci 2006; 47: 4138-4145.

29. Falsey RR, Marron MT, Gunaherath GM, Shirahatti N, Mahadevan D, Gunatilaka AA et al. Actin microfilament aggregation induced by withaferin $A$ is mediated by annexin II. Nat Chem Biol 2006; 2: 33-38

30. Vanden Berghe W, Sabbe L, Kaileh M, Haegeman G, Heyninck K. Molecular insight in the multifunctional activities of Withaferin A. Biochem Pharmacol 2012; 84: 1282-1291.

31. Hoe KK, Verma CS, Lane DP. Drugging the p53 pathway: understanding the route to clinical efficacy. Nat Rev Drug Discov 2014; 13: 217-236.

32. Lehmann S, Bykov VJ, Ali D, Andrén $\mathrm{O}$, Cherif $\mathrm{H}$, Tidefelt $\mathrm{U}$ et al. Targeting p53 in vivo: a first-in-human study with p53-targeting compound APR-246 in refractory hematologic malignancies and prostate cancer. J Clin Oncol 2012; 30: 3633-3639.

33. Maas AM, Bretz AC, Mack E, Stiewe T. Targeting p73 in cancer. Cancer Lett 2013; 332 229-236.

34. Candi E, Agostini M, Melino G, Bernassola F. How the TP53 family proteins TP63 and TP73 contribute to tumorigenesis: regulators and effectors. Hum Mutat 2014; 35 : 702-714.

35. D'Alessandro A, Marrocco C, Rinalducci S, Peschiaroli A, Timperio AM, Bongiorno-Borbone $L$ et al. Analysis of TAp73-dependent signaling via omics technologies. J Proteome Res 2013; 12: 4207-4220.

36. Tomasini R, Tsuchihara K, Wilhelm M, Fujitani M, Rufini A, Cheung CC et al. TAp73 knockout shows genomic instability with infertility and tumor suppressor functions. Genes Dev 2008; 22: 2677-2691.

37. Rufini A, Niklison-Chirou MV, Inoue S, Tomasini R, Harris IS, Marino A et al. TAp73 depletion accelerates aging through metabolic dysregulation. Genes Dev 2012; 26 : 2009-2014.

38. Cai Y, Qiu S, Gao X, Gu SZ, Liu ZJ. IASPP inhibits p53-independent apoptosis by inhibiting transcriptional activity of p63/p73 on promoters of proapoptotic genes. Apoptosis 2012; 17: 777-783.

39. Katayama H, Wang J, Treekitkarnmongkol W, Kawai H, Sasai K, Zhang $\mathrm{H}$ et al. Aurora kinase-A inactivates DNA damage-induced apoptosis and spindle assembly checkpoint response functions of p73. Cancer Cell 2012; 21: 196-211.

40. Zawacka-Pankau J, Kostecka A, Sznarkowska A, Hedström E, Kawiak A. p73 tumor suppressor protein: a close relative of p53 not only in structure but also in anti-cancer approach? Cell Cycle 2010; 9: 720-728.

41. Lau LM, Nugent JK, Zhao X, Irwin MS. HDM2 antagonist Nutlin-3 disrupts p73-HDM2 binding and enhances p73 function. Oncogene 2008; 27: 997-1003.

42. Bell HS, Dufes C, O'Prey J, Crighton D, Bergamaschi D, Lu X et al. A p53-derived apoptotic peptide derepresses p73 to cause tumor regression in vivo. J Clin Invest 2007; 117: 1008-1018.

43. Kravchenko JE, llyinskaya GV, Komarov PG, Agapova LS, Kochetkov DV, Strom E et al. Small-molecule RETRA suppresses mutant p53-bearing cancer cells through a p73dependent salvage pathway. Proc Natl Acad Sci USA 2008; 105: 6302-6307.

44. Sznarkowska A, Maleńczyk K, Kadziński L, Bielawski KP, Banecki B, Zawacka-Pankau J. Targeting of p53 and its homolog p73 by protoporphyrin IX. FEBS Lett 2011; 585: 255-260.

45. Issaeva N, Bozko P, Enge M, Protopopova M, Verhoef LG, Masucci M et al. Small molecule RITA binds to p53, blocks p53-HDM-2 interaction and activates p53 function in tumors. Nat Med 2004; 10: 1321-1328.

46. Shi Y, Nikulenkov F, Zawacka-Pankau J, Li H, Gabdoulline R, Xu J et al. ROS-dependent activation of JNK converts p53 into an efficient inhibitor of oncogenes leading to robust apoptosis. Cell Death Differ 2014; 21: 612-623.

47. Hahm ER, Moura MB, Kelley EE, Van Houten B, Shiva S, Singh SV. Withaferin A-induced apoptosis in human breast cancer cells is mediated by reactive oxygen species. PLoS One 2011; 6: e23354.

48. Bunz F, Dutriaux A, Lengauer C, Waldman T, Zhou S, Brown JP et al. Requirement for p53 and p21 to sustain G2 arrest after DNA damage. Science 1998; 282: 1497-1501.

49. Shelton $P$, Jaiswal AK. The transcription factor NF-E2-related factor 2 (Nrf2): a protooncogene? FASEB J 2013; 27: 414-423.

50. Gong JG, Costanzo A, Yang HQ, Melino G, Kaelin WG Jr, Levrero $M$ et al. The tyrosine kinase c-Abl regulates p73 in apoptotic response to cisplatin-induced DNA damage. Nature 1999; 399: 806-809.

51. Bernassola F, Salomoni P, Oberst A, Di Como CJ, Pagano M, Melino G et al. Ubiquitindependent degradation of p73 is inhibited by PML. J Exp Med 2004; 199: 1545-1557. 
52. Conforti F, Sayan AE, Sreekumar R, Sayan BS. Regulation of p73 activity by posttranslational modifications. Cell Death Dis 2012; 3: e285.

53. Caputo F, Vegliante R, Ghibelli L. Redox modulation of the DNA damage response. Biochem Pharmacol 2012; 84: 1292-1306.

54. Itoh K, Wakabayashi N, Katoh Y, Ishii T, Igarashi K, Engel JD et al. Keap1 represses nuclea activation of antioxidant responsive elements by Nrf2 through binding to the amino-terminal Neh2 domain. Genes Dev 1999; 13: 76-86.

55. Chen C, Yu R, Owuor ED, Kong AN. Activation of antioxidant-response element (ARE) mitogen-activated protein kinases (MAPKs) and caspases by major green tea polyphenol components during cell survival and death. Arch Pharm Res 2000; 23: 605-612.

56. Wade M, Li YC, Wahl GM. MDM2, MDMX and p53 in oncogenesis and cancer therapy. Nat Rev Cancer 2013; 13: 83-96.

57. Andreotti V, Ciribilli Y, Monti P, Bisio A, Lion M, Jordan J et al. p53 transactivation and the impact of mutations, cofactors and small molecules using a simplified yeast-based screening system. PLoS One 2011; 6: e20643.

58. Rossi M, Sayan AE, Terrinoni A, Melino G, Knight RA. Mechanism of induction of apoptosis by p73 and its relevance to neuroblastoma biology. Ann N Y Acad Sci 2004; 1028: 143-149.

59. Rufini A, Agostini M, Grespi F, Tomasini R, Sayan BS, Niklison-Chirou MV et al. p73 in cancer. Genes Cancer 2011; 2: 491-502.

60. Agostini M, Tucci P, Killick R, Candi E, Sayan BS, Rivetti di Val Cervo P et al. Neuronal differentiation by TAp73 is mediated by microRNA-34a regulation of synaptic protein targets. Proc Natl Acad Sci USA 2011; 108: 21093-21098.

61. Wong SW, Tiong KH, Kong WY, Yue YC, Chua CH, Lim JY et al. Rapamycin synergizes cisplatin sensitivity in basal-like breast cancer cells through up-regulation of p73. Breast Cancer Res Treat 2011; 128: 301-313.

62. Tian Z, D'Arcy P, Wang X, Ray A, Tai YT, Hu Y et al. A novel small molecule inhibitor of deubiquitylating enzyme USP14 and UCHL5 induces apoptosis in multiple myeloma and overcomes bortezomib resistance. Blood 2014; 123: 706-716.
63. Alard A, Fabre B, Anesia R, Marboeuf $C$, Pierre $P$, Susini $C$ et al. $N A D(P) H$ quinoneoxydoreductase 1 protects eukaryotic translation initiation factor $4 \mathrm{Gl}$ from degradation by the proteasome. Mol Cell Biol 2010; 30: 1097-1105.

64. Stewart SA, Dykxhoorn DM, Palliser D, Mizuno H, Yu EY, An DS et al. Lentivirus-delivered stable gene silencing by RNAi in primary cells. RNA 2003; 9: 493-501.

65. Szulc J, Wiznerowicz M, Sauvain MO, Trono D, Aebischer P. A versatile tool for conditional gene expression and knockdown. Nat Methods 2006; 3: 109-116.

66. Hedström E, Eriksson S, Zawacka-Pankau J, Arnér ES, Selivanova G. p53-dependent inhibition of TrxR1 contributes to the tumor-specific induction of apoptosis by RITA. Cell Cycle 2009; 8: 3576-3583.

67. Kawiak A, Piosik J, Stasilojc G, Gwizdek-Wisniewska A, Marczak L, Stobiecki M et al. Induction of apoptosis by plumbagin through reactive oxygen species-mediated inhibition of topoisomerase II. Toxicol Appl Pharmacol 2007; 223: 267-276.

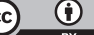

Cell Death and Disease is an open-access journal published by Nature Publishing Group. This work is licensed under a Creative Commons Attribution 4.0 International Licence. The images or other third party material in this article are included in the article's Creative Commons licence, unless indicated otherwise in the credit line; if the material is not included under the Creative Commons licence, users will need to obtain permission from the licence holder to reproduce the material. To view a copy of this licence, visit http://creativecommons.org/licenses/by/4.0

Supplementary Information accompanies this paper on Cell Death and Disease website (http://www.nature.com/cddis) 